\title{
AVALIAÇÃO DA UMIDADE NA COMPACTAÇÃO DO SOLO SUBMETIDO AO TRÁFEGO DE MÁQUINAS DE COLHEITA FLORESTAL ${ }^{1}$
}

\author{
Eduardo da Silva Lopes², Jean Alberto Sampietro³, Kátia Cylene Lombardi² e Andréa Nogueira Dias²
}

\begin{abstract}
RESUMO - Este trabalhou avaliou o efeito da umidade na compactação do solo submetido ao tráfego de máquinas de colheita de madeira em povoamentos de Pinus taeda L.. O estudo foi realizado nas áreas operacionais de uma empresa florestal localizada em Santa Catarina. Os dados foram obtidos em um Cambissolo Húmico em diferentes períodos, visando contemplar variações de umidade do solo. Foram comparadas a situação sem tráfego (ST) e aquela após o tráfego das máquinas (AT), por meio da determinação da densidade do solo (Ds) e da porosidade total (Pt), nas profundidades de 0 a 15, 15 a 30 e 30 a $50 \mathrm{~cm}$, e da resistência do solo à penetração (RP) no intervalo de profundidade entre 0 e $80 \mathrm{~cm}$. Os resultados mostraram que as maiores alterações provocadas pelo tráfego das máquinas nas propriedades físicas avaliadas ocorreram com o aumento da umidade. Foram também detectadas alterações em todo o perfil de solo. Isso demonstrou que a colheita da madeira realizada em solo úmido causa maior compactação. Dentre os modelos comparados para a estimativa da resistência do solo à penetração, o que teve o melhor ajuste foi o Modelo por Stepwise Aritmético com Variáveis Mistas (MSAM), que estimou a RP pela Ds inversa e a Ug de forma pura, explicando 71\% da variação dessa propriedade, e deixando em evidência a possível influência de outros fatores na estimativa da compactação do solo.
\end{abstract}

Palavras-chave: Compactação, Umidade e Colheita florestal.

\section{EVALUATION OF MOISTURE CONTENT ON COMPACTION OF SOIL SUBMITTED TO FOREST HARVESTING MACHINE TRAFFIC}

\begin{abstract}
This study evaluated the moisture effect in soil compaction submitted to wood harvesting machine traffic in Pinus taeda L. stands. The study was conducted in the operational areas of a forestry company located in Santa Catarina. The data were obtained in a Humic Cambisol soil in different periods in order to contemplate variations in soil moisture. It was compared the situation without traffic (WT) and after machine traffic (AT) by determining the bulk density (Ds) and soil total porosity (Pt) at the depths of 0 to 15, 15 to 30 and 30 to $50 \mathrm{~cm}$, and soil penetration resistance $(P R)$ in the depths between 0 to $80 \mathrm{~cm}$. The results showed that the major changes caused by traffic of machines in the physical properties occurred with increasing of moisture content. It was also detected changes in the entire soil profile. This demonstrated that the wood harvesting when performed in soils with high moisture contents will cause greater compaction. Among the models compared to estimate the soil penetration resistance, Stepwise Arithmetic Model with Mixed variables (MSAM), which estimated the RP by inverse Ds and $\mathrm{Ug}$ in pure form was the one with the best adjustement, explaining $71 \%$ of the variation of this property, and evidencing the possible influence of other factors in the estimation of soil compaction.
\end{abstract}

Keywords: Compaction, Moisture and Wood harvesting.

\footnotetext{
${ }^{1}$ Recebido em 25.09.2009 e aceito para publicação em 18.04.2011.

${ }^{2}$ Universidade Estadual do Centro-Oeste, UNICENTRO, Brasil. E-mail: < eslopes@pq.cnpq.br>e < katia.lombardi@pq.cnpq.br>.

${ }^{3}$ Universidade Federal de Santa Maria, UFSM, Brasil. E-mail: <engsampietro@gmail.com>.
} 


\section{INTRODUÇÃO}

A mecanização das operações de colheita de madeira intensificou-se no início da década de 90 com a abertura do mercado à importação. Entretanto, o aumento do tráfego de máquinas de grande porte vem impactando o solo de maneira considerável, provocando alterações nas suas propriedades físicas, químicas e biológicas, e afetando o seu potencial produtivo (DIAS JÚNIOR, 1999).

Segundo Seixas (1988), a compactação consiste na agregação das partículas do solo, reduzindo o volume por elas ocupado. Trata-se da tensão aplicada sobre o solo e mudanças resultantes em termos de aumento da densidade, decréscimo no volume de macroporos, infiltração e movimento interno de água mais lento e maior resistência mecânica do solo ao crescimento das raízes. Soane e Ouwerkerk (1994) dizem que a compactação é o processo de aumento na densidade aparente do solo, com incremento da resistência à penetração e redução na porosidade total e permeabilidade.

O efeito da compactação do solo no desenvolvimento das plantas é percebido quando a raiz encontra resistência mecânica ao seu crescimento. Segundo Camargo (1983), esse impedimento ocorre quando o diâmetro da raiz é superior ao poro no solo. Se a raiz não romper esse impedimento, todo o sistema radicular ficará denso e raso. Lockaby e Vidrine (1984) constataram reduções no crescimento em altura de uma floresta de Pinus taeda, variando entre 40 e $60 \%$, apesar da compactação do solo ter-se restringido aos $5 \mathrm{~cm}$ próximos à superfície. Worrel e Hampson (1997), ao avaliarem o crescimento de árvores em solos compactados pelo tráfego de máquinas florestais, constataram que o crescimento em altura foi reduzido de 13 a 50\% e o volume na ordem de 10 a 20\%. Borges (1986), avaliando o desenvolvimento do sistema radicular do Eucalyptus grandis em casa de vegetação, relatou que as raízes apresentaram dificuldades de crescimento com valores de densidade na faixa de 1,25 a $1,35 \mathrm{~g} \mathrm{~cm}^{-3}$.

Há diversos fatores que influenciam diretamente na compactação do solo, podendo destacar o tipo e a textura do solo; o teor de matéria orgânica; a presença de resíduos florestais sobre o solo; o teor de umidade; o tipo de máquinas e rodados; o tamanho, a forma e pressão de ar dos pneus, e o número de passadas da máquina (PORTERFIELD; CARPENTER, 1986; WARKOTSCH et al., 1994).
Seixas e Souza (2007) verificaram que as primeiras passadas das máquinas de colheita florestal foram responsáveis pelo maior incremento da compactação do solo, onde observaram que, em torno de $80 \%$ da compactação, foram resultantes das cinco primeiras passadas de um Forwarder. Já Fernandes et al. (1997) constataram que a influência do número de passadas dos rodados do Forwarder no nível de compactação de um solo ocorreu depois da primeira passada, voltando a ocorrer após a nona passada.

Makkonen (1989) afirma que o tráfego das máquinas sobre camadas de resíduos florestais também contribui para a redução do nível de compactação do solo. Seixas et al. (1995) detectaram reduções significativas ao redor de $40 \%$ do incremento na densidade de um solo, quando o tráfego de um Forwarder ocorreu sobre uma camada de resíduos da colheita de madeira.

Outro fator importante que pode influenciar na compactação refere-se à umidade. A condição de umidade no momento em que o solo está sendo compactado tem grande influência na redução e redistribuição do espaço poroso. Solos secos são mais resistentes a mudanças na distribuição do tamanho dos poros, e essa resistência é reduzida com o aumento do conteúdo de água (EAVIS, 1972). Alguns autores relatam que, com o aumento da umidade do solo, ocorre a maior lubrificação das partículas, atingindo os limites plásticos, onde a compactação se torna mais crítica. Com o conteúdo de água acima da capacidade de campo, o aumento da umidade resulta em uma redução da densidade, podendo causar a perda da estruturação original do solo (SILVA, 1984).

Lima et al. (2001) avaliaram a resistência do solo à penetração em trilhas entre linhas de plantio de eucalipto, em função da profundidade e da umidade no solo. O sistema de colheita avaliado foi composto por um Feller-buncher com pneus diagonais e um Skidder com pneus de baixa pressão. Segundo esses autores, a resistência do solo à penetração variou após o tráfego dos equipamentos e a variação mais acentuada ocorreu na profundidade de 0 a $10 \mathrm{~cm}$, sendo crescente com o aumento da profundidade.

Devido aos inúmeros fatores que afetam a compactação do solo, torna-se necessária a realização de estudos para o melhor entendimento dos fatores que explicam esse processo, possibilitando subsidiar o planejamento para a execução das operações da colheita florestal de forma eficiente e ambientalmente correta.

Revista Árvore, Viçosa-MG, v.35, n.3, Edição Especial, p.659-667, 2011 
Este trabalho teve por objetivo avaliar o efeito da umidade na compactação do solo submetido ao tráfego de máquinas de colheita da madeira em povoamento de Pinus taeda L. e desenvolver modelos para estimativa da compactação.

\section{MATERIAL E MÉTODOS}

\section{1. Área de Estudo}

O trabalho foi realizado nas áreas operacionais de colheita da madeira, em povoamentos de Pinus taeda $L$., pertencentes a uma empresa florestal, localizada no Estado de Santa Catarina. O clima da região de estudo é classificado, de acordo com Köppen, como mesotérmico úmido (Cfb), com temperatura média anual de $19,7^{\circ} \mathrm{C}$, precipitação média anual entre 1.600 e 1.700 mm, altitude de 530 m, latitude de $26^{\circ} 42^{\prime}$ '52' S e longitude de $49^{\circ}$ 29’00’'W (GAPLAN, 1986).

A área de estudo foi utilizada no passado para fins de produção agropecuária, havendo campos de pastagem antes de ser incorporada ao sistema de produção florestal. Os povoamentos de Pinus taeda L. apresentavam 35 anos de idade na ocasião da colheita da madeira, com 442 árvores por hectare, área basal de 45,82 $\mathrm{m}^{2} \mathrm{ha}^{-1}$, volume médio de $654,35 \mathrm{~m}^{3} \mathrm{ha}^{-1}$, sendo o peso médio individual de cada árvore de 0,89 t.

O solo da área foi classificado como um Cambissolo Húmico Aluminíco típico (CHa) de textura franco-argilo-arenosa, com horizonte A húmico e relevo de plano a suave ondulado, com 244, 116 e $640 \mathrm{~g} \mathrm{~kg}^{-1}$ de argila, silte e areia, e teor de carbono orgânico de 21,03 $\mathrm{g} \mathrm{dm}^{-3}$, 20,25 g dm d $^{-3}$ 18,31 $\mathrm{g} \mathrm{dm}^{-3}$, nas camadas de 0 a 15, 15 a 30 e 30 a $50 \mathrm{~cm}$ de profundidade, respectivamente.

\subsection{Sistema de colheita de madeira}

O sistema de colheita da madeira utilizado pela empresa era de Árvores Inteiras (Full Tree). A derrubada era realizada por um trator florestal Feller-buncher marca Caterpillar e modelo 522, com peso operacional de 30,4 t, com rodados de esteiras. A extração era realizada por trator florestal Skidder, marca Caterpillar e modelo 545 , com peso operacional de 18,2 t, tração 4 x 4 e rodados de pneus (pressão de $290 \mathrm{kPa}$ ) recobertos com semiesteiras na parte dianteira.

\subsection{Coleta de dados}

A coleta dos dados foi realizada após a colheita da madeira, sendo feita em diferentes períodos durante as condições secas e chuvosas, de acordo com a precipitação pluviométrica, procurando contemplar distintas umidades no solo.

A amostragem foi feita por meio da instalação de parcelas amostrais de $10 \times 25 \mathrm{~m}$, alocadas ao longo da linha de tráfego do Feller-buncher e do Skidder, procurando abranger diferentes intensidades de tráfego, contudo sem haver controle do tráfego, mantendo-se também as mesmas condições de relevo, com a finalidade de diminuir a variabilidade natural do solo. Em cada parcela, foram coletadas amostras de solo com estrutura preservada, buscando-se concentrar nos sulcos formados pela passagem dos rodados das máquinas após tráfego, e em locais próximos, distante 2 m da linha de tráfego, em solo sem tráfego. Para cada situação, as amostras foram coletadas em cinco pontos equidistantes $4 \mathrm{~m}$ entre si e em três níveis de profundidade ( 0 a 15, 15 a 30 e 30 a $50 \mathrm{~cm}$ ).

Foram instaladas e amostradas 14 parcelas, sendo realizada a coleta dos dados em local sem tráfego (ST) e após o tráfego (AT), totalizando 30 amostras por parcela (cinco pontos amostrais x três profundidades $\mathrm{x}$ dois locais de coleta). Em seguida, procedeu-se à classificação dos dados para a análise de diferentes classes de umidade gravimétrica do solo. A distribuição dos dados nas classes de umidade gravimétrica foi realizada em função do pareamento dos dados, correspondentes ao local sem tráfego (ST) e após tráfego (AT), por local de amostragem dentro da parcela. Ao final, obtiveram-se as seguintes classes de umidade: Ug1 (Ug < 0,30 kg kg-1); Ug2 $\left(0,30 \leq \mathrm{Ug}<0,40 \mathrm{~kg} \mathrm{~kg}^{-1}\right)$; $\mathrm{Ug} 3\left(\mathrm{Ug} \geq 0,40 \mathrm{~kg} \mathrm{~kg}^{-1}\right)$, totalizando 140 amostras por classe de umidade gravimétrica.

A densidade do solo (Ds), a porosidade total (Pt) e a umidade gravimétrica (Ug) foram determinadas conforme metodologia proposta pela EMBRAPA (1997), sendo coletadas amostras de solo com estrutura preservada com anéis volumétricos de $100 \mathrm{~cm}^{3}$. A resistência do solo à penetração (RP) foi determinada nos mesmos pontos amostrais, com o uso de um penetrógrafo eletrônico digital, marca EIJKELKAMP e modelo 06.15.SA, equipado com uma ponta em forma de cone com ângulo de vértice de $60^{\circ}$ e base de $1 \mathrm{~cm}^{2}$, com a velocidade de penetração controlada 
automaticamente em $2 \mathrm{~cm} \cdot \mathrm{s}^{-1}$. As leituras foram realizadas em intervalos de $1 \mathrm{~cm}$ até a profundidade de $80 \mathrm{~cm}$, conforme metodologia da ASAE citada por Balastreire (1987).

\subsection{Análises Estatísticas}

O delineamento estatístico utilizado foi o de experimento inteiramente casualizado. Para as variáveis de densidade do solo (Ds) e a porosidade total (Pt), as análises foram realizadas para as profundidades de 0 a 15, 15 a 30 e 30 a 50 cm, enquanto para a resistência à penetração (RP), embora as medições tenham sido feitas em intervalos de $1 \mathrm{~cm}$, as análises foram realizadas a cada $10 \mathrm{~cm}$ até a profundidade de $80 \mathrm{~cm}$.

Os valores de todas as variáveis foram avaliados pela diferença entre a situação sem tráfego (ST) e após tráfego (AT), pareados por local de amostragem dentro da parcela, sendo primeiramente submetidos ao teste de Bartlett, para verificação da homogeneidade das variâncias. Verificada a homogeneidade de variâncias, foi realizada em seguida a análise de variância e das médias comparadas pelo teste de Tukey, a 5\% de significância

Simultaneamente, foram desenvolvidas e ajustadas equações de regressão múltipla por Stepwise, sendo usadas como variáveis independentes a densidade do solo (Ds) e a umidade gravimétrica do solo (Ug), e, como variável dependente, a resistência do solo à penetração (RP). Para a avaliação estatística das equações, foram utilizados os coeficientes de determinação ajustado ( $\mathrm{R}^{2}$ aj) (DRAPER;SMITH, 1981), o erro-padrão da estimativa absoluto e em percentagem (Syx e Syx \%), e o valor de F.

\section{RESULTADOS}

\subsection{Densidade do solo}

A Tabela 1 mostra os valores médios de densidade do solo (Ds) nas diferentes classes de umidade gravimétrica e profundidade. Como pode ser visto, o incremento na densidade causado pelo tráfego das máquinas foi maior com o aumento da umidade do solo. Nota-se que, em condição mais seca (Ug1), quase não houve efeito do tráfego sobre a densidade, havendo incremento desse atributo na maior profundidade, ou seja, de 30 a $50 \mathrm{~cm}$.

Na classe de umidade gravimétrica Ug3 (Ug $\geq 0,40$ $\mathrm{kg} \mathrm{kg}^{-1}$ ), ocorreram os maiores incrementos na densidade do solo em todas as profundidades avaliadas. $\mathrm{Na}$ profundidade de 0 a $15 \mathrm{~cm}$, o tráfego das máquinas ocasionou incremento na densidade da ordem de 18,72\%, havendo diferença significativa em relação às condições de solo mais seco (Ug1 e Ug2), observando-se também diferença entre Ug2 e Ug1. No intervalo de profundidade entre 15 e 50 cm, não houve diferença entre as classes de maior umidade (Ug2 e Ug3), apesar de, na classe Ug3, terem ocorrido os maiores incrementos em densidade (17,63 e 16,35\%) para 15 a $30 \mathrm{~cm}$, e 30 a $50 \mathrm{~cm}$ de profundidade, respectivamente.

\subsection{Porosidade total}

Em termos de porosidade total (Tabela 2), verifica-se que a maior redução dessa propriedade também ocorreu na classe de umidade gravimétrica acima de $0,40 \mathrm{~kg} \mathrm{~kg}^{-1}$ (Ug3), com reduções de 9,44, 9,35 e 5,68\% nas profundidades de 0 a 15, 15 a 30 e 30 a 50 cm, respectivamente. A partir desse resultado, fica evidente a elevada relação da porosidade com a densidade do solo.

Tabela 1 - Valores médios de densidade do solo (Ds) nas diferentes classes de umidade gravimétrica e profundidade. Table 1 - Mean values of bulk density (Ds) in different classes of gravimetric moisture and depth.

\begin{tabular}{|c|c|c|c|c|c|c|c|c|c|}
\hline \multirow{4}{*}{$\begin{array}{l}\text { Classes de Umidade } \\
\text { Gravimétrica } \\
\left(\mathrm{kg} \mathrm{kg}^{-1}\right)\end{array}$} & \multicolumn{9}{|c|}{ Profundidade $(\mathrm{cm})$} \\
\hline & \multicolumn{3}{|c|}{ 0-15 } & \multicolumn{3}{|c|}{ 15-30 } & \multicolumn{3}{|c|}{$30-50$} \\
\hline & $\mathrm{Ds}(\mathrm{AT})$ & Ds (ST) & $\Delta \mathrm{Ds}$ & $\mathrm{Ds}(\mathrm{AT})$ & Ds (ST) & $\Delta \mathrm{Ds}$ & $\mathrm{Ds}(\mathrm{AT})$ & Ds (ST) & $\Delta \mathrm{Ds}$ \\
\hline & \multicolumn{3}{|c|}{$\mathrm{g} \mathrm{cm}^{-3}$} & \multicolumn{3}{|c|}{$\mathrm{g} \mathrm{cm}^{-3}$} & \multicolumn{3}{|c|}{$\mathrm{g} \mathrm{cm}^{-3}$} \\
\hline Ug1 (Ug < 0,30) & 1,29 & 1,31 & $-0,01 c$ & 1,38 & 1,38 & $0,00 \mathrm{~b}$ & 1,39 & 1,30 & $0,09 \mathrm{~b}$ \\
\hline Ug2 $(0,30 \leq \mathrm{Ug}<0,40)$ & 1,23 & 1,11 & $0,12 \mathrm{~b}$ & 1,26 & 1,08 & 0,16 a & 1,28 & 1,09 & 0,18 a \\
\hline Ug3 (Ug $\geq 0,40)$ & 1,05 & 0,85 & 0,20 a & 1,06 & 0,87 & 0,19 a & 1,10 & 0,92 & 0,18 a \\
\hline
\end{tabular}

Médias seguidas pela mesma letra minúscula na coluna não diferem estatisticamente entre si pelo teste de Tukey a 5\%; $\Delta$ : diferença considerando situação após tráfego e sem tráfego.

Revista Árvore, Viçosa-MG, v.35, n.3, Edição Especial, p.659-667, 2011 
Tabela 2 - Valores médios de porosidade total (Pt) nas diferentes classes de umidade gravimétrica e profundidade. Table 2 - Mean values of total porosity (Pt) in different classes of gravimetric moisture and depth.

\begin{tabular}{|c|c|c|c|c|c|c|c|c|c|}
\hline \multirow{4}{*}{$\begin{array}{l}\text { Classes de Umidade } \\
\text { Gravimétrica } \\
\left(\mathrm{kg} \mathrm{kg}^{-1}\right)\end{array}$} & \multicolumn{9}{|c|}{ Profundidade $(\mathrm{cm})$} \\
\hline & \multicolumn{3}{|c|}{$0-15$} & \multicolumn{3}{|c|}{$15-30$} & \multicolumn{3}{|c|}{$30-50$} \\
\hline & $\operatorname{Pt}(\mathrm{ST})$ & $\operatorname{Pt}(\mathrm{AT})$ & $\Delta \mathrm{Pt}$ & $\operatorname{Pt}(\mathrm{ST})$ & $\operatorname{Pt}(\mathrm{AT})$ & $\Delta \mathrm{Pt}$ & $\operatorname{Pt}(\mathrm{ST})$ & $\operatorname{Pt}(\mathrm{AT})$ & $\Delta \mathrm{Pt}$ \\
\hline & \multicolumn{3}{|c|}{$\mathrm{m}^{3} \mathrm{~m}^{-3}$} & \multicolumn{3}{|c|}{$\mathrm{m}^{3} \mathrm{~m}^{-3}$} & \multicolumn{3}{|c|}{$\mathrm{m}^{3} \mathrm{~m}^{-3}$} \\
\hline Ug1 $(\mathrm{Ug}<0,30)$ & 0,389 & 0,478 & $-0,089 \mathrm{c}$ & 0,369 & 0,462 & $-0,093 \mathrm{~b}$ & 0,414 & 0,439 & $-0,026 b$ \\
\hline $\mathrm{Ug} 2(0,30 \leq \mathrm{Ug}<0,40)$ & 0,533 & 0,514 & $0,019 \mathrm{~b}$ & 0,544 & 0,514 & 0,030 a & 0,552 & 0,505 & 0,047 a \\
\hline Ug3 (Ug $\geq 0,40)$ & 0,626 & 0,567 & $0,060 \mathrm{a}$ & 0,612 & 0,555 & $0,057 \mathrm{a}$ & 0,598 & 0,564 & $0,034 \mathrm{a}$ \\
\hline
\end{tabular}

Médias seguidas pela mesma letra minúscula na coluna não diferem estatisticamente entre si pelo teste de Tukey, a $5 \%$. $\Delta$ : diferença considerando condição sem tráfego e após tráfego.

\subsection{Resistência do solo à penetração}

A resistência do solo à penetração (RP) nas diferentes classes de umidade gravimétrica e profundidades estudadas é apresentada na Figura 1. Como pode ser visto, a resistência do solo à penetração apresentou um comportamento decrescente com o aumento da umidade dos solos. Foi ainda possível notar que, em todas as condições estudadas, o tráfego das máquinas de colheita da madeira causou alterações na resistência à penetração mesmo nas maiores profundidade do solo.

O comportamento da resistência à penetração mostra que, na classe de umidade acima de $0,40 \mathrm{~kg} \mathrm{~kg}^{-1}$ (Ug3) ocorreram as maiores diferenças em todas as profundidades avaliadas. Em nenhum intervalo de profundidade foram observadas grandes diferenças na classe de umidade abaixo de 0,30 kg kg-1 (Ug1), reforçando o conceito sobre os efeitos da umidade no momento da compactação.

$\mathrm{Na}$ classe de umidade gravimétrica abaixo de 0,30 $\mathrm{kg} \mathrm{kg}^{-1}$ (Ug1), houve valores acima de $2 \mathrm{MPa}$ nas primeiras camadas de profundidade, observando também na classe Ug2 nas maiores profundidades. A partir desse limite, o crescimento radicular começa a se tornar restritivo (RP crítica). Entretanto, esse valor aumentou, chegando a exceder $3 \mathrm{MPa}$ nas últimas camadas da classe Ug1, sendo considerado o limite para o desenvolvimento do sistema radicular de espécies florestais (RP restritiva).

É possível observar que os maiores incrementos na compactação do solo causado pelas máquinas de colheita da madeira ocorreram na situação de solo com as maiores umidades, chegando a atingir valores superiores a $40 \%$, refletindo a influência da umidade do solo em reestruturar as partículas.

\subsection{Estimativa da resistência do solo à penetração}

Foram selecionados os seguintes modelos apresentados na Tabela 3. A primeira equação (MAVP) se caracterizou por estimar a RP usando os valores de Ds e Ug de forma pura, sem modificar a unidade dessas variáveis. A segunda equação (MSAM) se caracterizou por estimar a variável dependente (RP) pela Ds inversa, e a Ug de forma pura. A terceira equação teve como variáveis explicativas a Ds de forma inversa e a Ug potencializada. A última equação (MBLA) usada nas comparações é o modelo proposto por Busscher (1990) de forma linearizada e adaptada. Para o uso desse modelo, primeiramente foi realização sua linearização, usando o logaritmo neperiano, e, em seguida, trocou-se do modelo original a variável umidade volumétrica $\left(\theta, \mathrm{m}^{3} \mathrm{~m}^{-3}\right)$ pela variável umidade gravimétrica (Ug, $\left.\mathrm{kg} \mathrm{kg}^{-1}\right)$.

O ajuste das equações é apresentado na Tabela 4. Dentre os modelos testados, o modelo por Stepwise aritmético com variáveis mistas (MSAM) apresentou o melhor coeficiente de determinação ajustado $\left(R^{2}\right.$ aj) com 0,7134 , seguido pelo modelo aritmético com variáveis puras (MAVP) $\left(R^{2}\right.$ aj = 0,7115), e pelo MSLM $\left(R^{2}\right.$ aj $\left.=0,7085\right)$ (Tabela 4). O erro-padrão da estimativa absoluta e percentual para os dois modelos com melhor $\mathrm{R}^{2}$ aj (MSAM e MAVP), foi de 0,2874 MPa e 18,64\%, respectivamente, para o MSAM, e de 0,2883 MPa e $18,70 \%$, respectivamente, para o MAVP. Em relação ao valor de F, o MSAM $(\mathrm{F}=308,35)$ se mostrou superior ao $\operatorname{MAVP}(\mathrm{F}=305,55)$.

Em geral, considerando todas as avaliações estatísticas, o modelo por Stepwise aritmético com variáveis mistas (MSAM) se mostrou o melhor para a estimativa da resistência do solo à penetração, seguido pelo MAVP. Nota-se, ainda, que o modelo proposto por Busscher (1990) linearizada e adaptado (MBLA) 

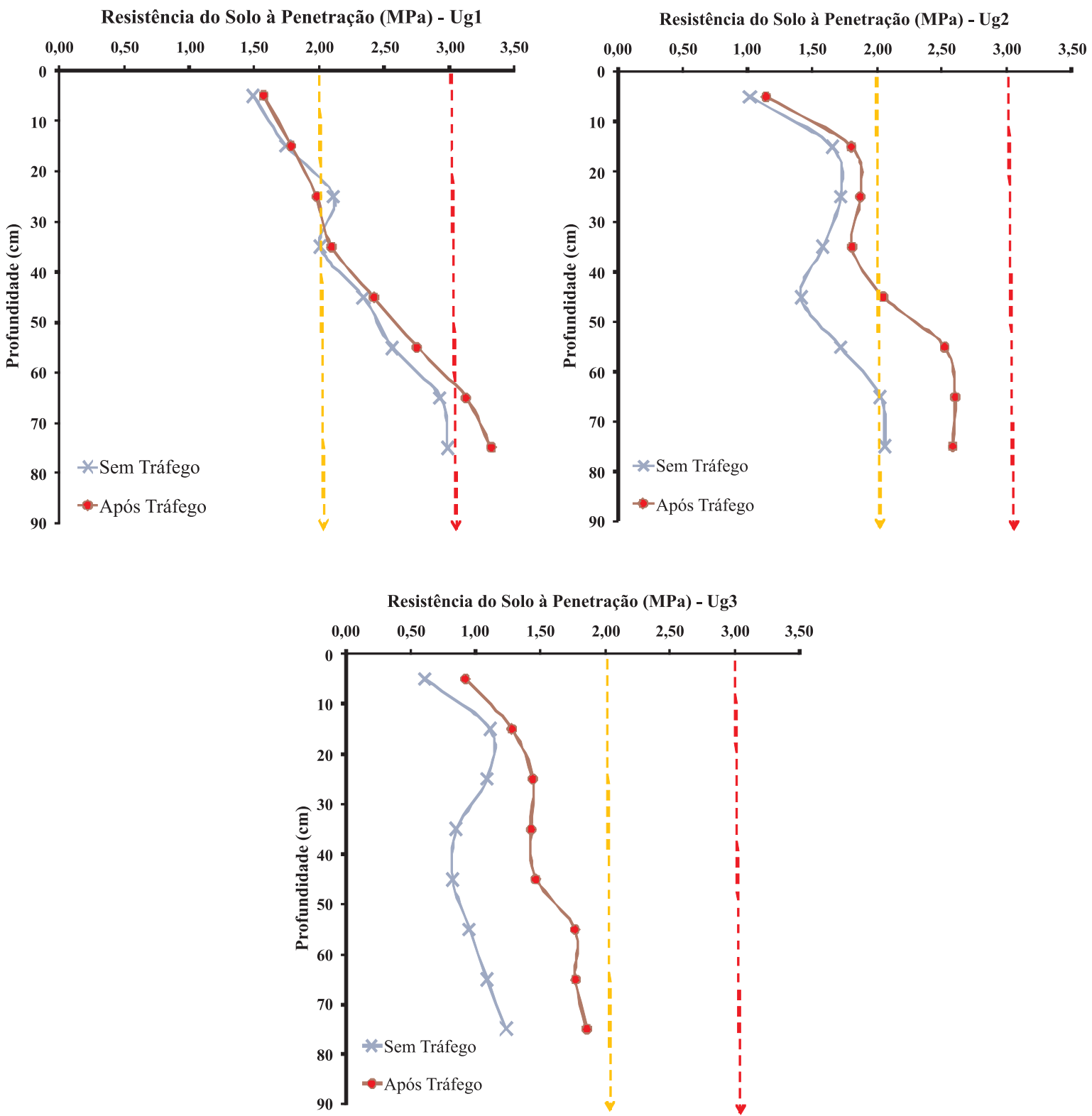

Figura 1 - Valores médios de resistência do solo à penetração (RP) sem tráfego (ST) e após tráfego (AT) para as diferentes classes de umidade gravimétrica; seta laranja indica valor de resistência à penetração crítica (RP crítica) e seta vermelha indica valor de resistência à penetração restritiva (RP restritiva).

Figure 1 - Mean values of soil penetration resistance (RP) without traffic (ST) and after traffic (AT) for different classes of gravimetric moisture; orange arrow indicates the value of critical soil penetration resistance (RP critical) and red arrow indicates the value of restrictive soil penetration resistance ( $R P$ restrictive).

foi o pior em todas as avaliações, salientando que em todas as estatísticas, para este modelo e para o MSLM, estas foram recalculadas para a variável de interesse, ou seja, para RP em MPa.
A Figura 2 ilustra a aplicação do modelo que teve melhor ajuste (MSAM), de acordo com os critérios escolhidos. É possível observar a variação da resistência do solo à penetração (RP estimada) em função da

Revista Árvore, Viçosa-MG, v.35, n.3, Edição Especial, p.659-667, 2011 
Tabela 3 - Modelos selecionados para estimativa da resistência do solo à penetração. Table 3 - Selected models to estimate soil resistance penetration.

\begin{tabular}{lcc}
\hline Siglas & Modelos & Tipos dos Modelos \\
\hline MAVP & $\mathrm{RP}=\beta_{0}+\beta_{1} \mathrm{Ds}+\beta_{2} \mathrm{Ug}$ & Modelo Aritmético com Variáveis Puras \\
MSAM & $\mathrm{RP}=\beta_{0}+\beta_{1} \mathrm{Ds}^{-1}+\beta_{2} \mathrm{Ug}$ & Modelo Stepwise Aritmético com variáveis Mistas \\
MSLM & $\mathrm{Ln} \mathrm{RP}=\mathrm{Ln} \beta_{0}+\beta_{1} \mathrm{Ds}^{-1}+\beta_{2} \mathrm{Ug}^{3}$ & Modelo Stepwise Logarítmico com variáveis Mistas \\
MBLA & $\mathrm{Ln} \mathrm{RP}=\operatorname{Ln} \beta_{0}+\beta_{1} \mathrm{Ln} \mathrm{Ug}+\beta_{2} \mathrm{Ln}$ Ds & Modelo de Busscher (1990) Linearizado e Adaptado \\
\hline
\end{tabular}

Ln: logaritmo neperiano; RP: resistência do solo à penetração; Ds: densidade do solo; Ug: umidade gravimétrica; $\mathrm{b}_{0}$, $\mathrm{b}_{1}$, $\mathrm{b}_{2}$ : coeficientes empíricos de ajuste.

Tabela 4 - Equações ajustadas para estimativa da resistência do solo à penetração. Table 4 - Adjusted equations to estimate soil resistance penetration.

\begin{tabular}{lcccccc}
\hline Modelo & Equações & $\mathrm{R}^{2}$ aj & Syx & Syx $(\%)$ & Valor F & $\mathrm{N}$ \\
\hline MAVP & $\mathrm{RP}=1,16^{* *}+1,33^{* *} \mathrm{Ds}-3,47^{* *} \mathrm{Ug}$ & 0,7115 & 0,2883 & 18,70 & 305,55 & 248 \\
MSAM & $\mathrm{RP}=4,21^{* *}-1,67^{* *} \mathrm{Ds}^{-1}-3,59^{* *} \mathrm{Ug}$ & 0,7134 & 0,2874 & 18,64 & 308,35 & 248 \\
MSLM & $\mathrm{Ln} \mathrm{RP}=1,91^{* *}-1,46^{* *} \mathrm{Ds}^{-1}-6,20^{* *} \mathrm{Ug}^{3}$ & 0,7085 & 0,2898 & 18,80 & 301,19 & 248 \\
MBLA & $\mathrm{Ln} \mathrm{RP}=-0,66^{* *}-0,72^{* *} \mathrm{Ln} \mathrm{Ug}+1,45^{* *} \mathrm{Ln} \mathrm{Ds}$ & 0,6129 & 0,3340 & 21,66 & 196,52 & 248 \\
\hline
\end{tabular}

*significativo a 5 \%; **significativo a 1 \%; Ln: logaritmo neperiano; RP: resistência do solo à penetração; Ds: densidade do solo; Ug: umidade gravimétrica; $\mathrm{R}^{2}$ aj: coeficiente de determinação ajustado; Syx: erro-padrão da estimativa em MPa; N: número de repetições.

umidade gravimétrica (Ug) e da densidade do solo (Ds). É possível verificar que, com a diminuição da umidade e aumento da densidade do solo, ocorrem os maiores valores de RP estimada.

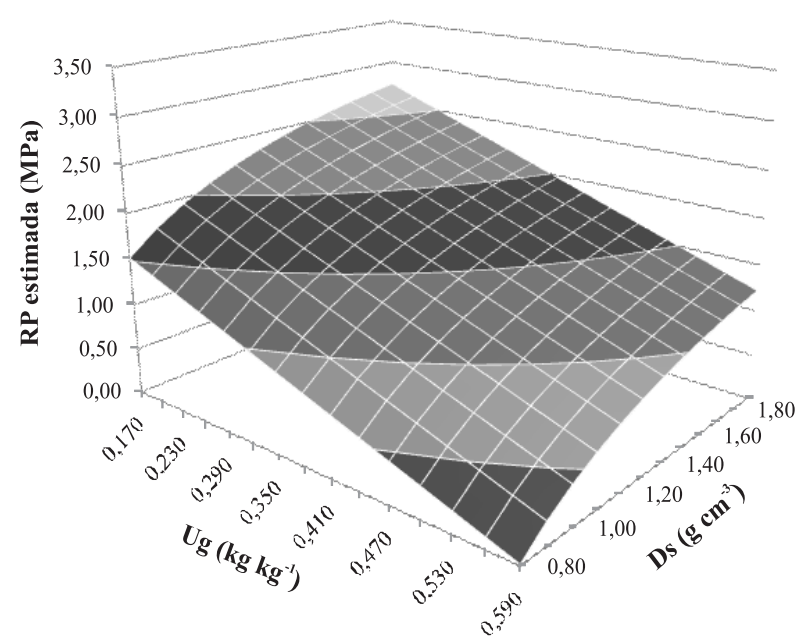

Figura 2 - Resistência à penetração estimada (RP estimada) pelo Modelo por Stepwise Aritmético com Variáveis Mistas (MSAM) em função da umidade gravimétrica (Ug) e densidade do solo (Ds).

Figure 2 - Penetration resistance (RP estimated) estimated by Stepwise Arithmetic Model with Mixed variables (MSAM) according to the gravimetric moisture (Ug) and bulk density (Ds).

\section{DISCUSSÃO}

É possível observar que, em todas as profundidades na classe de maior umidade gravimétrica (Ug3), houve incremento acima de $15 \%$ na densidade do solo, que, de acordo com Geist et al. (1989), pode restringir o crescimento radicular das plantas.

Os resultados mostram ainda que a porosidade se comportou semelhante à densidade do solo. Isso deixa em evidência a elevada relação dessas duas propriedades, conforme constatado por Seixas e Oliveira Júnior (2001). Assim, pode-se dizer que qualquer agente que pode alterar a porosidade tenderá a afetar também a densidade do solo.

De maneira geral, com maior umidade foram constatadas as maiores alterações em relação à densidade e porosidade total. Além disso, é importante enfatizar que susceptibilidade à compactação dos solos é fortemente dependente da quantidade de matéria orgânica e da textura do solo (LARSON; ALLMARAS, 1971; CLAYTON et al., 1987). O solo estudado apresentou elevado teor de areia e carbono orgânico em seu perfil, e esses teores podem ter influenciado favoravelmente a resistência às alterações provocadas pelo tráfego das máquinas de colheita da madeira.

Para a variável resistência do solo à penetração, os resultados confirmam as afirmações de que o conteúdo de água no momento em que o solo está sendo 
compactado tem grande influência na redução e redistribuição do espaço poroso. Os solos quando secos são mais resistentes às mudanças na distribuição do tamanho dos poros e sua resistência é aumentada com a redução do conteúdo de água. Portanto, as operações de colheita da madeira quando executada em solos com menores umidades sofrerão menor compactação em comparação aos solos úmidos.

É possível inferir que há a necessidade de um planejamento eficiente das operações de colheita da madeira quando realizadas em solos úmidos, com a adoção de medidas visando à minimização dos impactos ao meio ambiente e às rotações florestais futuras. Dentre as medidas, cita-se a restrição do tráfego das máquinas por toda a área, concentrando-a em carreadores prédeterminados ou o uso de máquinas com rodados de pneus extra largos de alta flutuação ou rodados de esteiras, que possibilitem a melhor distribuição do peso da máquina.

Para a estimativa da resistência do solo à penetração, embora tenha se conseguido bons ajustes, foi possível evidenciar outros fatores que também influenciam no comportamento dessa propriedade do solo, podendo ser como a textura e a matéria orgânica. A determinação da resistência do solo à penetração obtida no campo demonstrou a alta variabilidade dessa propriedade, pois é fortemente influenciada pelas condições do manejo do solo e pela intensidade de tráfego das máquinas, sendo dependente da umidade do solo.

O uso da modelagem estatística proporciona grandes evoluções em termos de exatidão e são válidos estudos dessa natureza, pois possibilitam melhor compreensão dos atributos que influenciam a compactação do solo.

\section{CONCLUSÕES}

De acordo com a análise e discussão dos resultados, as principais conclusões foram:

a) A umidade exerceu grande influência sobre a resistência do solo em relação ao tráfego das máquinas de colheita da madeira, e as maiores alterações nas propriedades físicas avaliadas ocorreram com o aumento da umidade do solo.

b) Houve alterações na resistência à penetração até $80 \mathrm{~cm}$ de profundidade, com valores acima de 2 e $3 \mathrm{MPa}$ em algumas situações. c) O melhor modelo ajustado para estimativa da resistência do solo à penetração explicou $71 \%$ da variação desta propriedade, mostrando que pode haver influência de outros fatores na estimativa dessa propriedade.

\section{AGRADECIMENTOS}

À CAPES e à Fundação Araucária, pelo apoio financeiro e a empresa florestal pela cessão da área de estudo.

\section{REFERÊNCIAS}

BALASTREIRE, L. A. Máquinas agrícolas. São Paulo: Manole, 1987. 307p.

BORGES, E. N. Resposta da soja e do eucalipto às camadas compactadas de solo. 1986. 54f. Dissertação (Mestrado em Solos e Nutrição de Plantas) - Universidade Federal de Viçosa, Viçosa, MG, 1986.

BUSSCHER, W. J. Adjustment of flat-tipped penetrometer resistance data to a commom water content. Transactions of the American Society of Agriculture Engineering, v.3, p.519-524, 1990.

CAMArgo, O. A. Compactação do solo e desenvolvimento de plantas. Campinas: Fundação Cargil, 1983. 44p.

CLAYTON, J. L.; KELLOGG, G.; FORRESTER, N. Soil disturbance and tree growth relations in central Idaho clear cuts. Intermountain Research Station. Forest Service. United States Department of Agriculture, 1987.

DIAS JÚNIOR, M. S. Avaliação quantitativa da sustentabilidade estrutural de um latossolo vermelhoamarelo situado em áreas experimentais da Cenibra: relatório do projeto de pesquisa. Lavras: Universidade Federal de Lavras, 1999. 23p.

DRAPER, N. R.; SMITH, H. Applied regression analysis. 2.ed. New York, John \& Wiley, 1981. 407p.

Revista Árvore, Viçosa-MG, v.35, n.3, Edição Especial, p.659-667, 2011 
EAVIS, B. W. Soil physical conditions affecting seedling root growth: 1- mechanical impedance, aeration, and moisture availability as influenced by bulk density and moisture levels in a sandy loam soil. Plant and Soil, v.36, p.613-622, 1972.

\section{EMPRESA BRASILEIRA DE PESQUISA}

AGROPECUÁRIA - EMBRAPA. Centro Nacional de Pesquisa de Solos. Manual de métodos de análise de solo. 2.ed. Rio de Janeiro: 1997. 212p.

FERNANDES, H. C.; ORLANDO, R. C.; LANÇAS, K. P. Compactação do solo em relação à exploração florestal: influência do "Forwarder” e do "Harvester". Engenharia na

Agricultura, v.5, n.4, p.276-280, 1997.

GAPLAN. Atlas de Santa Catarina. Aerofoto Cruzeiro, 1986.

GEIST, J. M.; HAZARD, J. W.; SEIDEL, K. W. Assessing physical conditions of some Pacific Northwest Volcanic ash soils after forest harvest. Soil Science Society of America Journal, v.53, p. 946-50, 1989.

LARSON, W. E.; ALLMARAS, R. P. Management factors and natural forces as related to compaction. In: Compaction in agricultural soils. St. Joseph: American Society of Agricultural Engineers Monograph, 1971. p.367-427.

LIMA, J. S. S. et al. Métodos geoestatísticos no estudo da resistência do solo à penetração em trilha de tráfego de tratores na colheita de madeira. Revista Árvore, v.32, n.5, p.931-938, 2008.

LOCKABY, B. G.; VIDRINE, C. G. Effect of logging equipment traffic on soil density and growth and survival of young loblolly pine. Southern Journal of Applied Forestry, v.8, n.2, p.109-112, 1984.

MAKKONEN, I. Choosing a wheeled shortwood forwarder. FERIC Technical Note, n.136, p.1-12, 1989.
PORTERFIELD, J. W.; CARPENTER, T. G. Soil compaction: An Index of potential compaction for agricultural tires. Transactions of the ASAE, v.29, n.4, p.917-922, 1986.

SEIXAS, F. Compactação do solo devido à mecanização florestal: causas, efeitos e práticas de controle. Circular técnica. IPEF, n.163, p.1-10, 1988.

SEIXAS, F.; OLIVEIRA JÚNIOR, E. D. Compactação do solo devido ao tráfego de máquinas de colheita de madeira. Scientia Forestalis, n.60, p.73-87, 2001.

SEIXAS, F.; SOUZA, C. R. Avaliação e efeito da compactação do solo, devido à freqüência de tráfego, na produção de madeira de eucalipto. Revista Árvore, v.31, n.6, p.1047-1052, 2007.

SEIXAS, F. et al. Effect of slash on forwarder soil compaction. In: COFE ANNUAL MEETING, 1995. Proceedings. Cashiers: COFE, 1995. p.77-86.

SILVA, A. P. Influência da compactação nas propriedades físicas do solo e no sistema radicular de plântulas de algodão (Gossypium hirsutum L.). 1984. 92 f. Dissertação (Mestrado em Solos e Nutrição de Plantas) - Escola Superior de Agricultura Luiz de Queiroz, Piracicaba, 1984.

SOANE, B. D.; OUWERKERK, C. Soil compaction problems in world agriculture. In: SOANE, B. D.; OUWERKERK, C. (Eds.) Soil compaction in crop production. Amsterdam: Elsevier, 1994. p.1-21.

WARKOTSCH, W. The impact of harvesting operation on timber quality: causes and remedies. South African Forestry Journal, n. 169, jun 1994.

WORREL, R.; HAMPSON A. The influence of some forest operations on the sustainable management of forest soils, a review. Forestry, v.70, n.1, p. 61-85, 1997. 
